\title{
Comunidades terapéuticas para madres drogadictas con hijos
}

\author{
Alessandra Simonelli, Vincenzo Calvo \\ Dipartimento di Psicologia dello Sviluppo e della Socializzazione. Universidad de Padova (Italia).

\section{RESUMEN}

Se revisa la experiencia italiana sobre comunidades terapéuticas con madres drogadictas con hijos que ha tenido lugar en diversas regiones italianas desde 1995. En concreto se estudian más profundamente 8 de estas comunidades del norte de Italia sobre las que se dispone de mayor información y sobre, algunas de ellas, se ha podido hacer observaciones directas frecuentes. Las comunidades pueden ser totalmente profesionales, religiosas o mixtas. La orientación teórica de los profesionales suele ser de tipo psicodinámico. Suele haber una combinación de terapias grupales e individuales. La estancia media de las madres suele ser de unos dos años. La experiencia parece ser positiva tanto para la madre como para el hijo, pero no existen estudios de seguimiento. Se plantean problemas a la hora de terminar la terapia y reinsertarse en la sociedad pues en ocasiones les cuesta abandonar el régimen residencial que han idealizado, por lo que algunas comunidades han desarrollado estructuras intermedias que facilitan la separación y el abandono de la comunidad terapéutica.

Palabras clave: madres drogadictas, comunidades terapéuticas, Italia, tratamiento.

\section{ABSTRACT}

The research reviews the Italian experience of therapeutic communities of drug-addicted mother with children, which have been in operation in several Italian regions since 1995. In particular, it makes a more in-depth study of 8 of these communities in northern Italy where there is more information available and where direct observations have been possible. The community can be totally professional, religious or mixed. The theoretical orientation of the professionals is generally of a psychodynamic kind. There is normally a combination of group and individual therapies. The average stay of mothers is generally two years. The experience seems to be positive for the mother and the child but there are no follow-up studies. Problems arise when the therapy ends and it is time for reinsertion in society since, on occasion, they find it difficult to leave the residential system, which they have idealised, so that some communities have developed intermediate structures that facilitate the separation and the departure from the therapeutic community.

Key words: drug addict mothers, therapeutic communities, Italy, treatment.
$\mathbf{E}$ n los últimos diez años se ha producido un cambio significativo en el enfoque con que se aborda el tratamiento de las situaciones de alto riesgo formadas por parejas de progenitor-hijo (o núcleos de madre-padre-hijo) en las que uno o ambos padres presentan cuadros de drogodependencia activa. Esta transformación, que puede apreciarse tanto a nivel institucional como privado, ha podido verificarse gracias a los esfuerzos llevados a cabo para pensar nuevas formas de actuar sobre este tipo de situaciones, nuevas formas que sintonicen mejor con el número y la complejidad de las circunstancias que, más o menos explícitamente, las rodean.

\section{PADRES DROGODEPENDIENTES E HIJOS}

\subsection{Mujeres y madres drogodependientes}

En el ámbito de la población drogodependiente la proporción de mujeres ha ido aumentando con los años, alcanzando, según datos recogidos en algunos estudios, un porcentaje del $30 \%$, frente al $15 \%$ que aparecía en los primeros trabajos sobre el tema (Aronica, 1987): de estos datos se deduce claramente que la posición de las mujeres en el mundo de la drogodependencia no es marginal, aunque la interpretación correcta de las cifras tenga que hacerse considerando 
que su exactitud es relativa por la discontinuidad y la falta de homogeneidad de la información disponible sobre el fenómeno. Por otra parte, lo que sí han planteado ha sido la necesidad de conocer mejor las facetas de la drogodependencia ligadas a la feminidad, y especialmente a lo "específicamente femenino", es decir, a las características de los síntomas y su etiopatogenia en la mujer. En este sentido, el objetivo es comprender las peculiaridades de los comportamientos y las vivencias internas que están en la raíz del malestar de la mujer drogodependiente.

Con este fin, diversos autores han intentado identificar factores evolutivos o condiciones de crecimiento que estén asociadas concretamente a la drogodependencia femenina. En los análisis del contexto familiar, se ha demostrado que uno de los elementos relevantes es la pertenencia a familias multiproblemáticas ligadas al mundo de la antisocialidad y del tráfico de drogas (Cancrini y La Rosa, 1991; Malagoli Togliatti y Rocchietta Tofani, 1987), familias que no están en condiciones de proteger a la joven del estrés emocional o relacional o de las diversas formas de marginación social típicas de estos contextos, sumergiéndola, por el contrario, plenamente, en esta red deprimida y deprimente. En estas familias las jóvenes crecen en unas condiciones de adultez y negligencia, viéndose implicadas precozmente en los "asuntos de familia" y en situaciones poco adecuadas para el crecimiento de un niño; la función protectora del núcleo familiar brilla por su ausencia, o está presente de forma poco adecuada (Aronica, 1987; cfr. Malagoli Togliatti y Mazzoni, 1993).

Otros trabajos evidencian dinámicas interpsicológicas e interpersonales descarriadas o conflictivas, caracterizadas por un enconamiento, frecuentemente con el progenitor del mismo sexo (Cancrini y Mazzoni, 1991; Todd y Selekman, 1989) que lleva a las jóvenes, al llegar a la adolescencia, a formas de pseudoindividuación, y a la manifestación de dificultades para adaptarse a los procesos que permiten alcanzar la autonomía personal y relacional (Tempesta, 1987). En estos casos, la drogodependencia puede aparecer como un síntoma con función homeoestática; es decir, un factor que mantiene el equilibrio del núcleo familiar, que cristaliza en torno a sus propios problemas (Tempesta, 1987).

Elementos comunes de las distintas interpretaciones que plantean los autores son las profundas vivencias de angustia y de confusión que transmiten estas mujeres, unas características que conforman su mundo interior y que transmiten a nivel de contratransferencia, mostrándose indefensas y, al mismo tiempo, manipuladoras, así como interiormente propensas a la violación de todo y de todos: esas características, que son las más evidentes pero no las únicas, están relacionadas con la falta de identificación con una imagen materna suficientemente buena (Winni- cott, 1975), sensible a la necesidad de seguridad que todo niño necesita (Ainsworth, Blehar, Waters y Wall, 1978; Ainsworth, Bell y Stayton, 1974) y que sintonice con ella (Stern, 1987), uno de los factores que garantizan el buen desarrollo afectivo-relacional del niño en las primerísimas fases de la vida. Lo cierto es que las madres drogodependientes se resisten a mostrar una semiótica positiva, dificultando uno de los aspectos más importantes tanto de la fase de diagnóstico como de tratamiento, sobre todo en situaciones de alto riesgo. Por eso es tan difícil potenciar factores protectores en estos casos en los que se puede hablar de "red social desgarrada" (Fava Vizziello, 1999), refiriéndose a la complicadísima situación existencial de estas personas. Sobre estos y sobre otros muchos aspectos se ha fundamentado, a lo largo del tiempo, el prejuicio de que los niños que son hijos de padres drogodependientes deben protegerse separándose de ellos (algunas veces definitivamente).

La realidad es que la mujer drogodependiente desea muchas veces un embarazo o un hijo para defenderse ante la vida o como un recurso para conseguir la salvación, aunque esta visión idealizada no impida que surjan fases de angustia ligadas a la colisión entre la evidencia y la siempre presente negación (consciente o inconsciente) del acontecimiento (Tempesta, 1987). Son síntomas de esta negación no reconocer el retraso menstrual como "señal" de embarazo, demorarse en verificarlo y, más tarde, dejar de atender las necesidades del feto. Por otra parte, el esfuerzo por crear una homeostasis psíquica se evidencia en la proyección de las partes buenas de la personalidad materna en el niño, con probabilidades de recuperación y de salvación (Di Giannantonio y Tempesta, 1985; Tempesta, 1987). Paralelamente, con mucha frecuencia la mujer embarazada continúa consumiendo drogas, manteniendo su estilo de vida sin modificar durante el mayor tiempo posible, siguiendo totalmente las pautas de una homeostasis toxicománica (Di Cagno y Lazzarini, 1984). También ocurre que algunas mujeres que han dejado de consumir drogas reanudan los hábitos drogodependientes en el parto y el post-parto, intentando sentirse a la altura de las circunstancias y calmar la sensación de culpa y de fallo (Fava Vizziello, Bullo y Simonelli, 1997; Tempesta y Pozzi, 1993).

El parto, de hecho, puede representar para la mujer una demostración brutal de lo que no ha sido capaz de hacer por su hijo y de la culpa que siente al enfrentarse a ello: cuando las exigencias de la realidad se hacen demasiado duras por dificultades del niño o de la madre, o por la ausencia de una red de apoyo, se rompe el sueño que se había concretado en el feto/hijo y pueden volver a surgir fuertes vivencias depresivas que hasta ese momento estaban contenidas porque se habían proyectado megalomaníaca- 
mente sobre el hijo y sobre una imagen materna idealizada (De Zordo, 1997; Tempesta, 1987).

Tanto si la mujer mantiene la abstinencia como si vuelve a iniciar el consumo de drogas, los problemas para desarrollar la función de madre surgen bastante precozmente y de modo evidente. En general, en estas madres se advierten dificultades para construir y mantener relaciones interpersonales gratificantes (con la pareja y con la familia de origen), una tendencia a adoptar un estilo de vida que lleva al aislamiento y, sobre todo, dificultades para reconocer y satisfacer las necesidades de su hijo (Fiks, Johnson y Rosen, 1985). A la incapacidad para desarrollar la función de madre, vivida de manera más o menos consciente, se unen otras dificultades recurrentes en muchos de estos casos: debilidad afectiva, pérdidas, separaciones, ausencia de continuidad afectiva en la familia de origen. Su forma de educar está condicionada por estas carencias, y suele caracterizarse por un exceso de implicación y actitudes autoritarias hacia el hijo, así como un rechazo de las influencias externas, tendencia al aislamiento, aceleración del proceso de autonomía del niño, control y rechazo de la comunicación. Además, la relación de estas madres con sus hijos suele estar cargada de ambivalencia, exigiéndose a menudo a los niños "hacerse cargo" de las expectativas y de los deseos de los padres y propiciando, de ese modo, una inversión de los roles y unas exigencias de adultez que se parecen mucho a lo que ellas mismas experimentaron en el pasado (Malagoli Togliatti, 1993).

Las dificultades de la madre y de la relación que mantiene con su hijo se manifiestan con más intensidad durante los primeros años de la vida del niño: la escasa autoestima, la ansiedad y la tendencia a la depresión que se identifican como principales características de la personalidad de estas mujeres, de hecho, produce en ellas una sensación de incapacidad para afrontar las responsabilidades maternas y el rol de madre (Fava Vizziello et al., 1997; Mazzoni, 1992).

\subsection{Los hijos de padres drogodependientes}

Junto al incremento numérico de las mujeres y, por lo tanto, de las madres, se asiste también a la "aparición" de niños que son hijos de padres drogodependientes: en el colegio, en los médicos de cabecera y en lugares de socialización y de acogida, además de en el ámbito de las instituciones que se hacen cargo de sus progenitores, la presencia de estos pequeños ha surgido como si, prácticamente de repente, un elemento latente se manifestase tras un periodo de aparente inexistencia. Además, la edad de estos niños es muy poco homogénea, y oscila entre los pocos meses de vida y la adolescencia (en algunos casos, también jóvenes-adultos): la amplitud de este periodo de tiempo evidencia indirectamente la dura- ción de su condición de niños no vistos, no observados, y por ello metidos en el interior de "burbujas protegidas", sin que se haya atendido a los factores de riesgo a los que estaban expuestos.

La presencia de hijos ha destruido progresivamente el mito (o el deseo no expresado) de la esterilidad generalizada de las parejas en las que al menos una de las dos partes es drogodependiente; en realidad, las mujeres drogodependientes se quedan embarazadas varias veces a lo largo de su vida: en los grupos estudiados por Aronica (1987) y por Palmieri (1991), por ejemplo, el porcentaje de mujeres embarazadas, con uno o más hijos, es del 50\%. La presunta dificultad para la procreación de esta población se atribuía bien a alteraciones neuroendocrinas provocadas por los efectos de algunas sustancias como la heroína y los opiáceos, bien a las inconstantes e irregulares condiciones de vida, a las carencias alimenticias y a los escasos hábitos higiénico-sanitarios: ninguno de estos factores, sin embargo, parecen limitar de modo significativo las posibilidades de que estas personas puedan tener hijos (Gennazzani, 1987).

Por otra parte, la presencia de los hijos ha hecho evidente la necesidad de comprender sus características y sus necesidades a distintos niveles: desde el punto de vista médico, estos niños suelen necesitar cuidados y atenciones específicas derivadas de las crisis de abstinencia, la seropositividad u otras patologías adquiridas durante el periodo de gestación (Zacchello y Giaquinto, 1997; Zuckerman y Brown, 1993), aunque en la mayoría de los casos estas dificultades sólo se limitan a los primeros años de su vida, y una vez superados remite la sintomatología. Por otra parte, en lo que respecta a su contexto afectivo-relacional durante el periodo de crecimiento, las necesidades, más o menos expresas, pueden ser de varios tipos: desde la ubicación física y la atención a las rutinas cotidianas básicas, a la idoneidad de los espacios afectivos, relacionales y contextuales ligados a su desarrollo.

\subsection{Sobre el padre, ese desconocido}

En los núcleos familiares descritos hasta este momento la postura de los padres siempre ha sido crítica: ocultos la mayor parte de las veces, contribuyen a proporcionar una imagen todavía más desgarrada e irrecuperable de estos casos, tanto en lo que respecta a la relación de pareja con la madre como en lo que concierne a la relación con el niño. Estas personas siguen siendo casi siempre drogodependientes activos que están totalmente inmersos en el circuito del tráfico, de los fraudes y de los sucesos "sucios" que rodean el mundo de de la drogodependencia y que tienen que ver con la desmesurada necesidad de dinero precisa para adquirir droga. Otros están en la cárcel, donde cumplen penas ligadas a las infraccio- 
nes que acabamos de citar, y frecuentemente siguen metidos en la senda de la drogodependencia. Algunos están enfermos, muchas veces en fases terminales de sida que no les permiten el desarrollo de ritmos de vida regulares, obligándoles a contínuos ingresos hospitalarios, a condiciones de precariedad física y existencial, a la imposibilidad de proyectar planes para la vida futura, ni como individuos ni como padres. Por último, en algunos casos, los padres han muerto a causa de la enfermedad y/o de la droga, o han recurrido al suicidio (frecuentemente por sobredosis) coincidiendo con el agravamiento de su estado físico o con circunstancias críticas de su vida.

Por lo tanto, en general, los padres se encuentran en unas condiciones de vida muy difíciles, que no les permiten proporcionar ningún tipo de apoyo ni a su pareja ni a su hijo.

\section{LA SITUACIÓN LEGAL DE LA PROTECCIÓN A LOS MENORES EN ITALIA}

Generalmente, los procedimientos legales que se adoptaban en materia de drogodependencia y paternidad, en los pocos casos que llegaban a conocimiento de los servicios, consistían en una indicación al Tribunal de Menores que implicaba, la mayoría de las veces, la suspensión o el revocamiento de la potestad de los progenitores hasta que al menos uno de ambos hubiese superado un periodo de prueba consistente en un tratamiento tutelado por los servicios encargados de la valoración del caso y de su evolución (Asesores Familiares, Ser. T, Servicios Sociales Municipales, Centros de Salud Mental, etc.). La aplicación de este tipo de procedimientos respetaba la exigencia primordial de proporcionar protección al menor en los casos en que las personas encargadas de su cuidado no estuviesen en condiciones de desarrollar adecuadamente dicha tarea, o cuando ellos mismos constituyesen una fuente de peligros para él.

El marco legal para los casos de incapacidad de los padres para ocuparse de los hijos se encuentra en los artículos 330, 332, 333 y 403 del Código Civil', en los que se indica la supresión de la potestad del progenitor cuando éste viola o descuida los deberes inherentes a ella con grave perjuicio para el hijo²; estos artículos se refieren a la necesidad de que el menor abandonado moral y materialmente por los adultos que deberían encargarse de su cuidado encuentre un lugar seguro (Ghezzi, 1996). Por todo ello, en la práctica, la exigencia de protección del menor se traduce en la necesidad de una valoración del contexto familiar en el que se encuentra. Concretamente, el mandato del Tribunal de Menores y, por lo tanto, el objeto de la averiguación está orientado a la evaluación de la capacidad de los padres para comprender al niño, para sintonizar con sus necesidades, así como a la comprensión de los motivos eventuales por los cuales no son capaces de atenderlo, para, finalmente, determinar los recursos afectivos y comportamentales que poseen para ejercer el papel de padres (Vadilonga, 1996). En estos casos, concretamente, nos encontramos frente a lo que diversos autores definen como una valoración coaccionada, en la que con "el término de coacción se entiende la utilización por parte de los terapeutas, en estrecha colaboración con los otros especialistas que ejercen la tutela del menor, de un mandato de valoración del Tribunal para los menores de edad para observar la relación entre el niño y sus padres y verificar si dicha relación (en la que se evidencia en el presente un perjuicio para el menor) puede llegar a evolucionar" (Vadilonga, 1996, pp. 8182). En general, el momento de la valoración es una fase en la que, junto a los elementos de riesgo para el niño, debe señalarse también la presencia de factores protectores que puedan contrastarse de alguna manera con los primeros: en este sentido, el riesgo y la protección se presentan como las dos caras de una misma moneda, y el peso de cada uno de estos factores y la conexión que existe entre ellos facilita la posible comprensión de la situación (Emiliani y Simonelli, 1997). Realizada de esta manera, la valoración contribuye a una definición de la función de la tutela del menor en un sentido psicológico, ya que prevé no sólo una perspectiva de eliminación de las condicio-

\section{(1) Artículos del Código Civil:}

Art.330: Pérdida de la potestad sobre los hijos- "El juez puede declarar la pérdida de la potestad cuando el progenitor viola o descuida los deberes inherentes a ella o abusa de los correspondientes poderes con grave perjuicio para el hijo. En tal caso, por motivos graves, el juez puede ordenar el alejamiento del hijo de la residencia familiar".

Art.332: Devolución de la potestad- "El juez puede devolver la potestad al progenitor que la ha perdido cuando, habiendo desaparecido las razones por las que se ha declarado la pérdida, ha quedado eliminado todo peligro de perjuicio para el hijo".

Art.333: Conducta del progenitor perjudicial para los hijos- "Cuando la conducta de uno o de ambos progenitores no sea causa que de lugar a la declaración de pérdida prevista en el art.330, pero se muestre de todos modos perjudicial para el hijo, el juez, de acuerdo con las circunstancias, puede adoptar los procedimientos convenientes y puede también disponer su alejamiento de la residencia familiar. Dichos procedimientos pueden revocarse en cualquier momento".

Art.403: Intervención de la autoridad pública a favor de los menores- "Cuando el menor se encuentra moral o meterialmente abandonado o criado en lugares insalubres o peligrosos, o bien por personas que por negligencia, inmoralidad, ignorancia o por otros motivos sean incapaces de hacerse cargo de su educación, la autoridad pública, por medio de los organismos de protección a la infancia, debe proporcionarle un lugar seguro hasta cuando se pueda proceder definitivamente a su protección".

(2) La cursiva es nuestra. 
nes presentes y objetivas de perjuicio para el pequeño, sino también un diagnóstico y pronóstico de su proceso de crecimiento afectivo-relacional, prestando una atención especial a la capacidad que tienen sus figuras de referencia para desarrollar la función de padres (Vadilonga, 1996).

\subsection{Soluciones experimentadas y limitaciones que han aparecido}

Una de las soluciones que más se solía adoptar durante el periodo de valoración era el alejamiento del niño de sus padres, si bien a través de procedimientos que evidenciaban tanto la ausencia de propuestas alternativas por parte de los servicios y de la red de acogida (aunque deba subrayarse la falta de homogeneidad entre distintas regiones y situaciones locales) como el prejuicio de que los padres drogodependientes son incapaces de proporcionar a sus hijos la protección adecuada, sobre todo si son muy pequeños (Pomodoro, 1993, 1996). El alejamiento del niño se realizaba a través de su ingreso en casas-familia de primera acogida o bien mediante programas de entregas y/o adopciones extra-familiares, en función de las posibilidades que existían en la zona y estaban a disposición de los especialistas. De todos modos, en los casos en los que al menos una de las familias de origen parecía estar en condiciones de proporcionar garantías de bienestar al pequeño, lo más frecuente era que se entregase a los abuelos, aunque por otra parte esta solución también ha sido la que, en función de la proporción de éxitos obtenidos, ha suscitado una controversia mayor. La elección de la familia paterna o materna como lugar de inserción del niño se ha considerado un fracaso precisamente haberse utilizado como medida generalizada y prácticamente automática, que si bien puede funcionar como respuesta inmediata a la protección que los niños necesitan, no supone para los padres ninguna fuente de motivación ni les estimula para recuperarse (Cirillo, 1996; cfr. Fava Vizziello y Stocco, 1997; Ghezzi, 1996). Lo cierto es que en muchos casos la incapacidad de las madres para atender a sus hijos parece un mensaje dirigido a sus propios padres. De hecho, estas poblaciones están formadas por mujeres jóvenes que llegan a la drogodependencia como consecuencia de una relación profundamente insatisfactoria con la madre o con el padre y la madre, que se perciben afectivamente ausentes (Cirillo, 1997). En este contexto de relaciones afectivas, la madre drogodependiente puede llegar a utilizar a su hijo (que ya no es un niño salvador, una vez que los cuidados concretos han situado en la realidad las expectativas mágicas que se habían depositado en él) para resarcirse de sus propias carencias a través de los cuidados que le proporcionen sus padres. Concretamente, lo que madre busca es resarcirse en este eje femenino (madre, hija drogodependiente, hijo). Pero "este tipo de estrategia está abocada al fracaso. El fallo más obvio se produce cuando la abuela no responde a la llamada, resistiéndose a ocuparse de las necesidades del nieto de la misma manera que a las anteriores demandas de ayuda por parte de la hija, de este modo provocativo y culpabilizante. (...) Pero el fracaso de la estrategia (...) se produce también cuando la abuela se muestra disponible. De hecho, muchas veces el cariño que ésta desarrolla hacia el nieto, al que ve como un regalo que le ha dado la vida y que le permite vivir una nueva experiencia de maternidad, se traduce en una ulterior traición de la hija" (Cirillo, 1997, p. 201).

\section{LAS COMUNIDADES PARA MADRES DROGO- DEPENDIENTES CON HIJOS}

Todos estos factores, unidos a otros más, son los que han demostrado la necesidad de revisar las praxis habituales. Hay que tener en cuenta, además, las dificultades reales que rodean el hallazgo de familias idóneas o de estructuras adecuadas para la acogida de los niños, para la gestión de la relación con los padres y las familias de origen y para la coordinación de los múltiples servicios implicados. Paralelamente, existen experiencias piloto que han permitido a algunos padres drogodependientes desarrollar un proceso de rehabilitación personal manteniendo a la vez la relación con su hijo; se trata de actuaciones de apoyo que han llevado a éxitos positivos: este tipo de experiencias han favorecido posturas que se plantean la posibilidad real de que los padres drogodependientes puedan desarrollar adecuadamente su función y su relación con su hijo, como "espacio sano" de atención del niño, aunque sea en el contexto de una situación psicológica y social muy difícil (Fava Vizziello y Stocco, 1997).

El descubrimiento de una paternidad posible en personas con síntomas de drogodependencia ha generado necesidades de acogida y de atención en las que se apliquen parámetros nuevos; el aspecto más evidente es el que se refiere al cambio del sujeto clínico que es objeto de observación y de cuidado: el foco ya no es la persona, y su tratamiento tampoco se aborda de un modo sectorial y parcelado por parte de los individuos encargados, respectivamente, de la protección y la acogida del progenitor o del niño. Lo más importante ahora es la situación psico-social en toda su complejidad, intentando abordar los cuidados globalmente (cuidar a las personas y las relaciones entre ellas), así como la integración profesional entre los diversos entes y trabajadores. Este cuadro se constituye a nivel clínico como una entidad nueva, que ya no está formada por progenitores con síntomas de drogodependencia, sino por madres o padres con hijos (y a veces por familias enteras) que deben someterse a 
un proceso en el que se diagnostiquen tanto los factores de riesgo como las posibilidades de rehabilitación, y para ellos debe proyectarse un proceso tutelado que favorezca el cambio.

Las reflexiones que siguen se basan en un trabajo de observación y recogida de datos en el que han participado 8 comunidades para madres drogodependientes con hijos, situadas en diversas regiones del norte de Italia: en 4 de ellas ha sido posible realizar un trabajo de observación directa del trabajo y de la vida en la comunidad, además de la organización general de la estructura (espacios, tiempos, modalidades de intervención, etc.), mientras que en las otras 4 se ha analizado el material descriptivo proporcionado por las propias comunidades (opúsculos, desplegables, material de divulgación, artículos, etc.).

En las comunidades en las que ha sido posible la observación directa, la recogida del material se ha realizado en un periodo de tiempo que oscila entre los 6 y los 12 meses, a través de las siguientes fuentes:

- Visitas directas a las comunidades (casas, habitaciones, lugares de recreo y de trabajo, etc.);

- Entrevistas con el personal encargado de la asistencia (voluntarios, trabajadores, psicólogos);

- Entrevistas con las mujeres residentes en las distintas estructuras;

- Participación en distintos momentos de la vida comunitaria (comidas, actividades laborales, reuniones con los usuarios, reuniones y supervisiones con los trabajadores, etc.);

- Recogida del material de divulgación producido por la comunidad (opúsculos, desplegables, material de divulgación, artículos, etc.);
Obviamente, en las comunidades en las que no ha sido posible llevar a cabo una observación directa, esta última fuente ha sido fundamental para la recogida de datos.

\subsection{La organización general de las Comunidades}

Las comunidades para madres drogodependientes con hijos son estructuras que ofrecen tutela residencial a parejas de madre-hijo (algunas veces también al padre), proponiéndoles un proceso de rehabilitación articulado. Son ya numerosas en el territorio nacional y comparten unas características específicas comunes, aunque también poseen cierta autonomía organizativa y de gestión. Por otra parte, cada región posee una normativa compleja y articulada que las define y encuadra, actuando como marco en el que se establecen sus roles, funciones y límites mediante múltiples parámetros de valoración (puestos disponibles, estructuras, cuotas mensuales, etc.).

En general, se trata de casas bastante grandes situadas en periferias urbanas o en pueblos, con amplios espacios internos y/o externos para poder desarrollar las distintas actividades comunitarias: cada una tiene una capacidad de acogida de hasta 10-12 parejas, con una edad para los niños que va desde pocos meses (algunas veces, sin embargo, se acogen también mujeres embarazadas) a los 6-7 años. Las parejas madre-hijo se alojan en habitaciones sencillas o dobles, de acuerdo con la disponibilidad de espacio y las exigencias del niño. La figura 1 muestra la estructura general de las comunidades estudiadas.

\section{Figura1. La estructura general de las comunidades para madres drogodependientes e hijos}

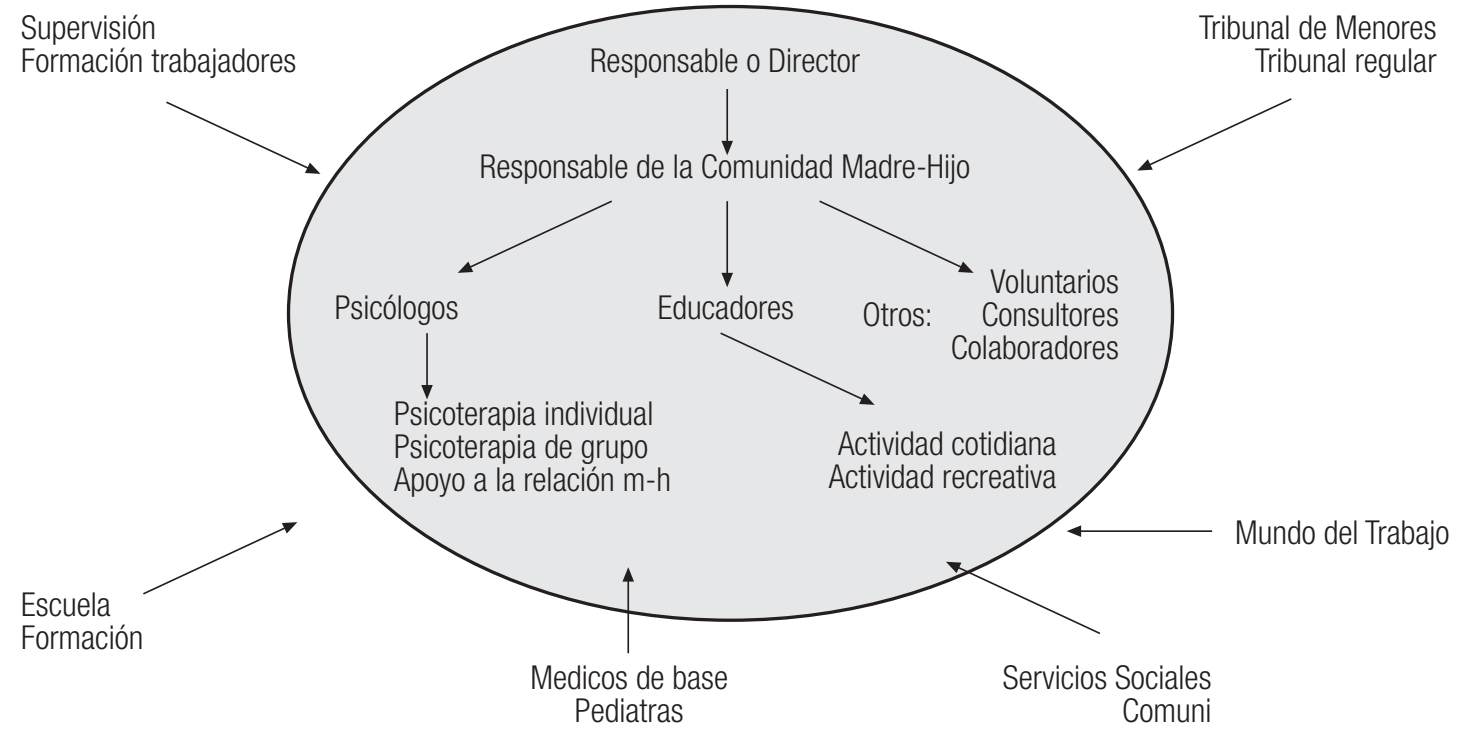




\subsection{Modelos y protagonistas de la actuación}

Los distintos tipos de comunidades existentes se distinguen entre sí en función del modelo y de la organización de la intervención; en general, las diferencias se pueden establecer en función de la matriz cultural y de la organización-formación del equipo de cuidadores. Estas dos líneas guía son fundamentales para la comprensión del trabajo que se desarrolla en el interior de las estructuras.

En lo relativo a la matriz cultural, se puede distinguir entre comunidades que podríamos definir como de inspiración religiosa y comunidades fundadas en una matriz laica. En lo que concierne a la organizaciónformación del equipo encargado de los cuidados, las estructuras se reparten entre comunidades gestionadas exclusivamente por personal religioso o voluntario; comunidades en las que el personal voluntario o religioso trabaja junto a profesionales y comunidades gestionadas exclusivamente por profesionales.

Uno de los ejemplos más representativos de comunidades de matriz cultural laica gestionada por profesionales es el que ofrecen las estructuras en cuyo ámbito trabajan exclusivamente psicólogos psicoterapeutas y educadores de comunidades con formación específica. Como base teórica y técnica, el modelo de referencia es un enfoque de orientación psicodinámica dirigido al estudio de la personalidad normal y patológica (Lis, Stella y Zavattini, 1999). Este tipo de organización está ligado a unos objetivos concretos y también a unas herramientas específicas para conseguir la rehabilitación, tanto en lo que concierne a las técnicas de intervención utilizadas (psicoterapia individual y de grupo, grupos de apoyo para las familias de origen, etc.) como a los profesionales que se eligen y la formación que se les exige.

Por el contrario, los modelos complejos están formados por comunidades en las que el origen y el seguimiento del proyecto de acogida de las madres drogodependientes con hijos es responsabilidad de trabajadores del Ser.T. (centro de tratamiento ambulatorio con psiquiatra y psicólogo) y de educadores de comunidades profesionales, que son los que se encargan de la atención psicológica y educativa de los casos (personal profesional); sin embargo, la estructura de acogida residencial se sitúa en el interior de una comunidad para jóvenes drogodependientes más amplia gestionada por religiosos (personal religioso). Esta ubicación crea cierta perplejidad en la esencia de la matriz cultural de la comunidad, religiosa en lo referente a la organización cotidiana y a la gestión general de algunos objetivos educativos, laica en lo que concierne a la actuación psicológica. Esta ambigüedad de fondo podría llegar a ser con el tiempo un factor de debilidad de la actuación, tanto en lo referente a los "mensajes" que se dirigen a los usuarios como en lo que respecta a la comunicación entre los profesiona- les y los voluntarios implicados en la gestión de los casos y en las decisiones relativas a la actuación propiamente dicha. Sin embargo, esta organización es lo que confiere su peculiaridad a este modelo comunitario y puede suponer un estímulo innovador para el tipo de atención que requieren algunos casos particulares, en el sentido de que la conexión entre la comunidad de madres con hijos con la de jóvenes drogodependientes varones permite la acogida de núcleos familiares completos (madre, padre e hijo) en el ámbito de un único contexto, facilitando la intervención sobre el padre y sobre la relación padre-hijo, que es un aspecto que se descuida en otras situaciones.

En algunas regiones se ha consolidado bastante un modelo de comunidad que, siendo de matriz cultural esencialmente religiosa, plantea una colaboración de manera sistemática y estable entre personal voluntario o religioso y personal con formación profesional. Los voluntarios laicos, en algunos casos, pueden haber sido también ex usuarios de la propia comunidad o padres de usuarios que permanecen ligados a la estructura y que siguen dispuestos a colaborar en el desarrollo de algunas de sus actividades (sobre todo de las recreativas).

Finalmente, también existen comunidades de matriz cultural religiosa y gestionadas exclusivamente por personal religioso o voluntario, vinculadas a la labor de apostolado de distintas órdenes religiosas y, por último, las experiencias de grupos-familia en las que familias de voluntarios acogen a las parejas de madre-hijo en sus propias casas, ofreciéndoles manutención y posibilidades para la rehabilitación.

Está claro que todos estos aspectos suponen también profundas diferencias en la concepción y en la realización de los programas de atención terapéutica y educativa, ampliando notablemente la tipología de las actuaciones y los factores que intervienen en el cambio.

Las modalidades de intervención psicológica que se ofrecen se basan todas en un acercamiento conjunto (o sea, sobre el progenitor y sobre el niño a la vez) y muy intensivo (gracias a la condición residencial de las parejas): en otras palabras, esas comunidades se caracterizan principalmente por la propuesta de un programa terapéutico-rehabilitativo que tiene como foco principal el sistema familia-progenitor-hijo considerado en toda su complejidad (Meisels, Dichtelmiller e Fong-Ruey Liaw, 1996; Stern, 1997). A pesar de todo, las estructuras se distinguen entre sí por diferencias relacionadas con lo que Stern (1997) define (a propósito de las terapias madre-hijo) como punto de entrada del terapeuta en el sistema progenitor-hijo. Los puntos de acceso terapéutico al sistema, de hecho, son los que en mayor medida parecen diferenciar las posibilidades teóricas y metodológicas de las actuaciones seguidas en cada una de las comunidades: en resumen, éstas se diferencian según el nivel 
de entrada preseleccionado para la intervención (nivel de comportamiento o nivel de la representación) y el sujeto principal de la misma (la madre, el hijo; Stern, 1997). En las comunidades analizadas, generalmente el sujeto preseleccionado para la intervención es la madre, y, junto a ella, la relación madre-hijo en aquellas estructuras que tienen previsto un apoyo psicológico en este sentido; en cambio, sólo se actúa sobre el hijo en casos de necesidad evidente, y los que intervienen son principalmente consultores externos contratados específicamente para ello (psicólogos clínicos especialistas en desarrollo infantil, neuropsiquiatras infantiles). Este tipo de estructuras parten de la premisa de la normalidad con la que los trabajadores están dispuestos a acoger niños: lo cierto es que en la mayoría de los casos se trata de pequeños física y psicológicamente sanos que se encuentran en un ambiente con una sintomatología que debe ser afrontada y resuelta.

La elección de un punto de acceso concreto diferencia las distintas estructuras entre sí y determina el nivel de la actuación: generalmente las comunidades fundadas sobre una matriz cultural de voluntariadoreligiosa y con personal exclusivamente voluntario enfocan la actuación prioritariamente hacia la modificación del comportamiento. En este contexto, el punto de acceso privilegiado es aquel que permita que cambien los comportamientos interactivos disfuncionales entre la madre y el hijo, desarrollándose sobre todo a través de actuaciones de naturaleza prevalentemente educativa. Paralelamente, en algunas comunidades nos encontramos frente a estructuras en las que distintos tipos de trabajadores desarrollan distintas técnicas de actuación (modificando el sistema desde puntos de vista diferentes) dentro del mismo programa terapéutico. Concretamente, en las comunidades con actuaciones proyectadas desde matrices culturales diversas y personal con distinta formación puede producirse una especie de separación entre los roles de los trabajadores y los puntos de entrada terapéutica en el sistema madre-hijo: los que se encargan de los aspectos educativos de la actuación prefieren el acceso a la modificación de los comportamientos, mientras que los terapeutas se concentran principalmente en el cambio del mundo representacional del progenitor, considerándolo una entrada favorable para la transformación de la relación con el niño.

Por otra parte, los elementos que integran el sistema familia-progenitor-hijo son dependientes entre sí y están en una interacción dinámica constante, por lo que, al margen de punto que se elija para acercarse y entrar en la terapia, los cambios que se producen en su interior están determinados por su propia naturaleza (Stern, 1997).

\subsection{Construir la motivación para el cambio}

El mecanismo que incita a las madres drogodependientes a ingresar en comunidades se puede definir como una especie de chantaje; en otras palabras, es como si se les hiciese la siguiente advertencia: "por ser drogodependiente no puedes ser madre, la ley y los organismos que se ocupan de ello te lo impiden y protegen a tu hijo, de ti incluida, hasta que no inicies un proceso que te permita ser madre a todos los efectos". Es frecuentemente el miedo a "perder el niño" (similar al de los primeros meses de embarazo, que probablemente las mujeres no sintieron en aquel periodo, quizás porque no eran conscientes de esta posibilidad, así como tampoco del propio embarazo) el estímulo que empuja al ingreso: si por una parte podría objetarse que es, ciertamente, una motivación extrínseca al significado de la "curación" (que en psicología se apoya en gran medida en el reconocimiento de una necesidad por parte de la persona con la que se trabaja: es decir, estoy sufriendo, hay algo que no funciona y quiero cambiarlo), por otra parte este miedo es, a nuestro juicio, quizás la primera emoción verdadera que surge en estas mujeres que llevan años alteradas y confusas, con el cuerpo y con los afectos anestesiados. Ese miedo, y también el descubrimiento de un sentimiento fuerte que les une al niño y que no creían poseer, posiblemente no lo han sentido durante el embarazo o en el parto, y no lo habrían descubierto nunca sin la amenaza de los procedimientos judiciales.

Lo cierto es que muchas veces las madres drogodependientes no son madres maltratadoras, abusadoras o violentas; son sobre todo madres que se ven frustradas de la cotidianidad de su rol como progenitoras y de la constancia y la estabilidad que requieren los niños, sobre todo en los primeros años de vida. Las madres drogodependientes son madres que se "olvidan" del niño, que lo "pierden" de su memoria y que, como no lo han tenido siempre presente, algunas veces lo "dejan" (ellas las primeras, sin esperar el mandato de los tribunales): lo dejan en la escuela (olvidándose de la hora del cierre), lo dejan Ilorar porque no saben qué es lo que quieren, lo dejan jugar solo porque no son capaces de inventar juegos.

\subsection{Duración de la actuación}

Las intervenciones psicoterapéuticas realizadas suelen combinar terapias de grupo o individuales y la mayor parte están dirigidas por psicoterapeutas o psicólogos clínicos con formación psicodinámica. Este planteamiento contempla un proceso de reelaboración (en el tiempo pasado en la comunidad y en otros tiempos de su existencia) de la carga patógena de algunos de los núcleos principales de la historia afectivo-relacional de la persona. El proceso de rehabilitación sigue un ritmo relacionado con la duración del 
periodo de residencia de la pareja, que se convierte en el escenario que estructura la rehabilitación y la terapia: la duración de la comunidad, de hecho, contempla un periodo de aproximadamente dos años (algunas veces se convierten en tres), que en algunos casos se subdivide en diferentes fases evolutivas, centrado en el rol que tiene la persona en el grupo de usuarios, en las responsabilidades que se les confían, en el mayor o menor contacto con el mundo exterior, etc. Se trata de aspectos que constituyen una parte muy importante de los elementos terapéuticos ligados a la reconstrucción que estas mujeres pueden llevar a cabo a través de la comunidad.

En primer lugar, se suele reconstruir el pasado remoto, dando mucha importancia a la historia personal y a las experiencias infantiles de estas madres, desprovistas de afectos, de seguridad y poco escuchadas cuando fueron niñas y plantearon sus necesidades. La desintoxicación de sustancias psicotrópicas y del tipo de vida asociado a ellas favorece, por otra parte, la reelaboración del pasado próximo y de las experiencias ligadas a la drogodependencia. Este pasado (que a veces ha tenido una duración de 150 20 años), ha supuesto la única alternativa que estas personas han podido encontrar para solucionar su malestar interno, y está formado no sólo de droga, sino también de cárcel, prostitución, maltratos: todos ellos sucesos traumáticos que necesitan ser repensados a la luz de las nuevas condiciones que están experimentando. De todos modos, el objetivo más importante de las comunidades es la construcción del presente, en todas sus diversas y complicadas facetas internas y comportamentales. Para ello la intervención psicológica se apoya y refuerza con otra de rehabilitación-educación, en una acción sinérgica de elaboración real y fantasiosa del malestar.

El proceso temporal seguido por las madres afecta también a los niños que, en edad muy precoz, en la que deben construirse y aprenderse muchas cosas, pasan un largo periodo en las comunidades. En el pasado, de hecho, ya durante el periodo de gestación los pequeños han estado expuestos a las drogas consumidas por la madre, a una alimentación frecuentemente irregular y a ritmos de vida fatigosos. Aunque no haya habido complicaciones durante el parto o los momentos inmediatamente posteriores, los primeros meses de vida de la mayoría de estos niños han estado sembrados de separaciones de la madre o de las personas más cercanas, y no es frecuente que se hayan respetado sus tiempos y sus exigencias. Otras veces, muchos de ellos han tenido que afrontar nuevos encuentros (que para un niño de pocos meses o de algunos años es una tarea bastante difícil): pensemos en las visitas a los médicos especialistas, en los contactos con asistentes sociales, psicólogos u otro tipo de personal de los servicios de asistencia o de las comunidades; en fin, algunos han sufrido modificacio- nes bruscas en las condiciones de vida cotidianas, como cambios de casa, de ciudad, etc.

Por esto, entre las actuaciones que se realizan sobre los niños en las comunidades destacan las que contribuyen a la construcción de condiciones de vida estables, tanto en sus aspectos afectivos profundos como en todo aquello que concierne a las prácticas y a las rutinas cotidianas. Este segundo punto se afronta en el momento en el que se produce el ingreso en las escuelas infantiles, o en la escuela, cuando el niño participa en actividades recreativas, deportivas y en otros momentos de socialización en el interior o en el exterior de las comunidades. Se trata de procesos que favorecen la socialización con los semejantes, y también con otras figuras adultas distintas de la madre y de las personas que trabajan en la comunidad, uno de los factores fundamentales para que se produzca la inserción completa de estos niños en un contexto vital que no esté estigmatizado por las dificultades y por las historias existenciales de sus padres. El objetivo es proporcionarles la posibilidad de una realización personal futura, a nivel escolar, laboral y social, a pesar de lo poco ventajoso del punto de partida.

En resumen, el trabajo desarrollado por la comunidad y en la comunidad tiene, como objetivo primordial, proporcionar a cada una de estas mujeres y a sus hijos una perspectiva para el futuro que comprenda su reinserción autónoma en el interior de una red reconstruida y reintegrada, al menos en lo relacionado con el riesgo de recaída y de daño para el menor. La proyección hacia el futuro es una necesidad extremadamente importante tanto para las personas implicadas (las madres y los hijos) como para las que trabajan en las comunidades.

\subsection{Comunidades: la función terapéutica de los lugares}

Otra característica de todas las comunidades es el hecho de que estén planteadas prioritariamente como lugares en los que las madres pueden estar junto a sus hijos, sitios protegidos en los que se puede desarrollar un apego físico y psicológico que desempeña múltiples funciones, favoreciendo sobre todo en la madre una importante regresión al rol de hija y niña necesitada (de los trabajadores, de los psicólogos y de todos los encargados de cuidarles): ese movimiento psíquico no está, ciertamente, desprovisto de ambivalencia por su valencia simbólica de enfrentamiento a nuevas figuras paternas o maternas, esta vez cercanas y atentas a las necesidades, pero también severas y exigentes cuando es necesario. La posibilidad de experimentar sentimientos mixtos al encontrarse con estos nuevos "progenitores" institucionales puede permitir a las mujeres encontrar los hilos relacionales y representacionales interrumpidos en su infancia y 
adolescencia, y permitirles revisar el pasado y sus contenidos mortíferos.

Esta situación permite por tanto considerar a la comunidad en conjunto como un ambiente paternal en el que se tutela y se apoya la maternidad de estas mujeres, en vez de limitarse a castigarla en sus facetas relacionadas con la inadaptación y la dificultad.

En lo que respecta a los niños, en todas las comunidades hay personas (educadores, psicólogos, neuropsiquiatras infantiles), que:

- tienen la preparación necesaria para proteger su derecho a permanecer y crecer con sus madres aunque no sean perfectas, porque piensan que los vínculos primarios (aunque se hayan olvidado en el transcurso de la vida) son de todas formas un patrimonio de cada uno, irrenunciable e indeleble;

- pueden protegerles de sus propias madres en los casos en los que la intervención falle, desembocando en el desgarro que supone la adopción y la separación definitiva del progenitor;

- pueden crear lugares en los que sea posible mantener una constancia del objeto y de las relaciones, es decir, en los que sea posible crecer sin que el mundo se derrumbe en pocos segundos, gracias a la continuidad de un contexto estable de vida (que también significa dormir todas las noches en la misma cama, tener los mismos juguetes y volver siempre a la misma casa después de la escuela);

Todo esto permite a los niños acogidos experimentar un mundo en el que los adultos hacen lo que dicen o se esfuerzan por hacerlo, en el que las madres no trabajan siempre fuera de la ciudad o están en el hospital (como muchos abuelos o familiares encargados de ellos se apresuran a responder cuando preguntan), en el que las madres están, son lo que son, pero están presentes.

\subsection{Factores de riesgo}

La observación prolongada y el estudio del material relativo al tiempo que los usuarios permanecen en comunidades ha evidenciado una característica compartida en los distintos tipos de estructuras que puede dibujarse como un posible factor de riesgo tanto para ellas como para las personas que acogen. Nos referimos al riesgo de caer en terapias interminables en el interior de contextos que podríamos definir como idealizados, inexistentes en el mundo real y que contrastan de tal manera con la brutalidad de las experiencias precedentes de estas mujeres, que generan unas expectativas mágicas de "inserción eterna" en comunidad.

Las dificultades de separación que experimentan tanto los usuarios como las personas que trabajan con ellos se han evidenciado sobre todo en las fases de inserción laboral en el exterior de las comunidades, en los primeros pasos que sitúan a la mujer en el contexto al que se deberá enfrentar cada vez con más frecuencia en el futuro. De la misma manera, los contactos con la realidad del niño (la escuela, otras familias, etc.) generan vivencias de desigualdad y de vergüenza en las madres, que la empujan de nuevo hacia la comunidad y la protección que esta representa. El rol de la comunidad como base segura (Ainsworth, et al., 1978), de hecho, es un objetivo deseado por todos los trabajadores, pero que también debe permitir la necesaria separación hacia el exterior, so pena de una acogida interminable y un estancamiento terapéutico.

En esta dirección (favorecer un tránsito hacia la separación que no sea brutal) se encuentran la estructuras intermedias que existen en algunas de las comunidades estudiadas: se trata de apartamentos situados en lugares cercanos a las comunidades y ligados a ellas por algunas actividades diarias y semanales que las madres y los hijos siguen desarrollando en ellas hasta el momento de la separación verdadera y propiamente dicha hacia otros lugares (la reinserción en el lugar de origen, traslados por motivos laborales, la elección de un lugar en el que vivir de una manera estable). Gracias a estos procesos la autonomía puede convertirse en una meta menos angustiosa y que provoque menos miedo, favorecida por el carácter gradual de la transición. Lo interesante es plantear que las comunidades "buenas" son las que son capaces de destruir gradualmente lo que han construido en el periodo de permanencia de la pareja que ha estado en ella: el trabajo de crear un vínculo entre la madre y el niño y la comunidad y entre ellos y el tipo de actuación que se ha dirigido hacia ellos se corresponde también con el del trabajo de favorecer, por parte de las personas que trabajan allí, una autonomía y una separación que confiera autenticidad a las metas alcanzadas. La madre y el niño tienen que enfrentarse necesariamente a la vida real de fuera de la comunidad, si no quieren caer de nuevo en los mecanismos de dependencia y de malestar sobre los que estas mujeres habían construido muchas de las dinámicas personales y familiares del pasado y gran parte de sus relaciones primarias. Por ello la tarea de las comunidades debe plantearse como una actuación focal, breve en el tiempo y orientada hacia la elaboración de una separación gradual entre la pareja de madre e hijo y la propia comunidad: una separación que proteja el crecimiento de ambos (la madre y el hijo) y que les permita recomponer y reanudar la retícula de su propia existencia. 


\section{BIBLIOGRAFÍA}

Ainsworth, M. D. S., Bell, S. M. y Stayton, D. J. (1974). Infant-Mother attachment and social development: Socialisation as a product of reciprocal responsiveness to signals. En M. P. M. Richards (Ed.). The Integration of a Child into a Social World (pp. 99-135). London: Cambridge University Press

Ainsworth, M. D. S., Blehar, M. C., Waters, E., y Wall, S. (1978). Patterns of Attachment. A Psychological Study of the Strange Situation. Hillsdale, NJ: Erlbaum.

Aronica, E. (1987). Il rapporto tra donna e droga. Alla ricerca di possibili differenze e specificità. Torino: CIC Edizioni Internazionali.

Cancrini, L. y La Rosa, C. (1991). Quei temerari sulle macchine volanti. Roma: Nuova Italia Scientifica.

Cancrini, L. y Mazzoni, S. (1991). Le tossicomanie: un'esperienza di ricerca. In N. Lalli (Ed.). Manuale di psichiatria e psicoterapia (pp. 249-269). Napoli: Liguori.

Cirillo, S. (1996). La tutela dei figli dei tossicodipendenti. En D. Ghezzi y F. Vadilonga (Eds.). La tutela del minore (219-234). Milano: Raffaello Cortina.

Cirillo, S. (1997). Tutela del figlio tossicodipendente: dinamica familiare e presa in carico. En G.M. Fava Vizziello y P. Stocco (Eds.). Tra genitori e figli la tossicodipendenza (pp. 47-61). Milano: Masson.

De Zordo, M.R. (1997) Genitori e bambini: genitori-bambini. En G.M. Fava Vizziello y P. Stocco (Eds.). Tra genitori e figli la tossicodipendenza (pp. 207-212). Milano: Masson.

Di Cagno, L. y Lazzarini, A. (1984). I/ neonato e il suo mondo relazionale. Roma: Borla.

Di Giannantonio, M. y Tempesta, E (1985). Farmacodipendenza e gravidanza. Bollettino Nazionale per le Farmacodipendenze e I'Alcoolismo, VIII, 1-3.

Emiliani, F. y Simonelli, A. (1997). Psicopatologia evolutiva e maltrattamento infantile. Psicologia Clinica dello Sviluppo, 1(3), 327-354.

Fava Vizziello, G.M. (1999). Interventi nella rete sociale lacerata. Convegno Internazionale. Università di Padova.

Fava Vizziello, G.M., Bullo, P. y Simonelli, A. (1997). I genitori tossicodipendenti e i loro figli. En G.M. Fava Vizziello y P. Stocco (Eds.). Tra genitori e figli la tossicodipendenza (pp. 103-120). Milano: Masson.

Fava Vizziello, G.M. y Stocco, P. (1997). Tra genitori e figli la tossicodipendenza. Milano: Masson

Fiks, K.B., Johnson, H.L. y Rosen, T.S. (1985). MethadoneMantained mothers: three-year follow-up of parental functionning. The Internationa Journal of Addictions, 20(5), 45-57.

Gennazzani, A.R. (1987). Riproduzione e farmacodipendenza. Bollettino Nazionale per le Farmacodipendenze e I'Alcoolismo, 6, 609-615.

Ghezzi, D. (1996). II bambino compromesso e la sua famiglia in difficoltà. En D. Ghezzi y F. Vadilonga (Eds.). La tutela del minore (pp. 1-28). Milano: Raffaello Cortina.
Lis, A., Stella, S. y Zavattini, G.C. (1999). Introduzione. En A. Lis, S. Stella y G.C. Zavattini (Eds.). Manuale di Psicologia Dinamica. Bologna: II Mulino.

Malagoli Togliatti, M. (1993). La tossicodipendente di fronte alla maternità e i suoi rapporti con la rete familiare. En M. Malagoli Togliatti y S. Mazzoni (Eds.). Gravidanza e tossicodipendenza (pp. 62-84). Milano: Giuffré.

Malagoli Togliatti, M. y Rocchietta Tofani, L. (1987). Famiglie multiproblematiche. Roma: Nuova Italia Scientifica.

Malagoli Togliatti, M. y Mazzoni, S. (1993). Maternità e tossicodipendenza. Milano: Giuffré.

Mazzoni, S. (1992). Tossicomania e gravidanza. En M. Ammaniti (Ed.). La gravidanza tra fantasia e realtà (pp. 217-235). Roma: II Pensiero Scientifico.

Meisels, S.J., Dichtelmiller, M. y Fong-Ruey Liaw (1996). Un'analisi multidimensionale dei programmi di intervento per la prima infanzia. En C.H. Zeanah (Ed.). Manuale di salute mentale infantile (pp. 317-339). Milano: Masson.

Palmieri, V. (1991). Osservazioni e analisi del tossicodipendente detenuto. Quaderni della Fondazione Villa Maraini, 1, Roma.

Pomodoro, L. (1993). Aspetti giuridici. En M. Malagoli Togliatti y S. Mazzoni (Eds.). Gravidanza e tossicodipendenza (pp. 13-24). Milano: Giuffré.

Pomodoro, L. (1996). Prefazione. En D. Ghezzi y F. Vadilonga (Eds.). La tutela del minore. Milano: Raffaello Cortina.

Stern, D.N. (1987). I/ mondo interpersonale del bambino. Torino: Bollati Boringhieri.

Stern, D.N. (1997). La costellazione materna. Torino: Bollati Boringhieri.

Tempesta, E. (1987). Storia psichica della gravida tossicodipendente. Bollettino Nazionale delle Farmacodipendenze e I'Alcoolismo, X, 601-607.

Tempesta, E. y Pozzi, G. (1993). Aspetti medico-epidemiologici della gravidanza nel corso della tossicodipendenza. En M. Malagoli Togliatti y S. Mazzoni (Eds.). Gravidanza e tossicodipendenza (pp. 25-38). Milano: Giuffré.

Todd, T.C. y Selekman, T. (1989). Family therapy approaches with adolescent substance abusers. New York: Gardner Press.

Vadilonga, F. (1996). Lo psicologo e la valutazione. En D. Ghezzi y F. Vadilonga (Eds.). La tutela del minore (pp. 71-94). Milano: Raffaello Cortina.

Winnicott, D.W. (1975). Dalla pediatria alla psicoanalisi. Firenze: Martinelli.

Zacchello, F. y Giaquinto, M. (1997). Figlio di madre tossicodipendente. Problemi clinici e assistenziali. En G.M. Fava Vizziello y P. Stocco (Eds.). Tra genitori e figli la tossicodipendenza (pp. 161-168). Milano: Masson.

Zuckerman, B. y Brown, E.R. (1996). Abuso materno di sostanze e sviluppo infantile. En C.H. Zeanah (Ed.). Manuale di salute mentale infantile (pp. 125-138). Milano: Masson. 
